\title{
HIDRÓliSE ÁCIDA DE CELULOSE ASSISTIDA POR ULTRASSOM PARA OBTENÇÃO DE FURFURAL
}

\author{
W. SANTOS ${ }^{1}$, D. SANTOS ${ }^{1}$, T. L. GATIBONI ${ }^{1}$, P. A. MELLO ${ }^{2}$ e E. M. M. FLORES ${ }^{2}$ \\ ${ }^{1}$ Universidade Federal de Santa Maria, Departamento de Engenharia Química \\ ${ }^{2}$ Universidade Federal de Santa Maria, Departamento de Química \\ paola.mello@ufsm.br
}

\begin{abstract}
RESUMO - A conversão de biomassa a produtos químicos possibilita a obtenção de polímeros, solventes e combustíveis, a partir de fontes renováveis de carbono. O presente estudo viabilizou o uso do ultrassom na intensificação da conversão de celulose para obtenção de furfural. Foram avaliados diferentes sistemas de aplicação de ultrassom (banhos, sondas e cup horns) com experimentos comparativos sob agitação mecânica e estudo da potência liberada em cada caso. Os seguintes parâmetros foram avaliados: amplitude (30, 50 e 70\%), temperatura de sonicação $\left(30,50\right.$ e $\left.70{ }^{\circ} \mathrm{C}\right)$ e concentração de $\mathrm{HNO}_{3}\left(4,6\right.$ e $\left.8 \mathrm{~mol} \mathrm{~L}{ }^{-1}\right)$. Os produtos foram quantificados por cromatografia a líquido de alta eficiência acoplada a espectrometria de massa por tempo-de-vôo (UPLC-ToF-MS) e a pureza foi confirmada por ressonância magnética nuclear (NMR, de ${ }^{13} \mathrm{C}$ e ${ }^{1} \mathrm{H}$ ). $\mathrm{O}$ resíduo após hidrólise foi avaliado por microscopia eletrônica de varredura (SEM). No sistema cup horn, com amplitude de $50 \%$, temperatura de $30{ }^{\circ} \mathrm{C}$ e 60 minutos de sonicação, utilizando $\mathrm{HNO}_{3} 4 \mathrm{~mol} \mathrm{~L}^{-1}$ foram obtidos os rendimentos reacionais mais elevados, chegando a $78 \%$ de conversão a furfural. A utilização de um ácido diluído volátil associado à temperatura amena de reação, representam vantagens significativas do processo assistido por ultrassom.
\end{abstract}

\section{INTRODUÇÃO}

A busca por matérias-primas alternativas para processos industriais tem sido alvo de diversos estudos. A utilização de fontes renováveis de carbono reduz a dependência de fontes fósseis para obtenção de produtos de química fina (Silveira, 2008). De acordo com a literatura, a disponibilidade de recursos fósseis tende a reduzir nos próximos anos, em função das dificuldades de extração e dos impactos negativos causados pelo seu uso em larga escala (Mothé e Miranda, 2010).

Dentre as potenciais fontes alternativas ao petróleo, a biomassa surge como um expoente em pesquisas tecnológicas. De maneira geral, a biomassa é formada por carboidratos, lignina, proteínas, gorduras e, numa menor extensão, por várias outras substâncias, tais como vitaminas, terpenos, carotenóides, alcalóides, pigmentos e flavorizantes (Rodrigues, 2011). Essa vasta composição proporciona a diversificação da matriz industrial, impulsionando a descentralização dos meios produtores de matérias-primas e locais de geração de energia, podendo resultar em um modelo industrial mais sustentável. 
O furfural é um importante produto químico usado na síntese de vários compostos sintéticos de alto valor agregado (álcool furfurílico, tetra-hidrofurano e ácido levulínico). Industrialmente, é produzido por processos de desidratação de açúcares, através de um complexo mecanismo reacional que envolve longos tempos reacionais, elevadas temperaturas e pressões (Rocha, 2015). As principais fontes de matéria-prima utilizadas nesses processos são plantas com alto teor de celulose, um biopolímero, formado pela união de moléculas de $\beta$ glicose. Esta é uma das mais abundantes fontes de energia renovável e que pode permitir a síntese de vários produtos em escala industrial (Barbosa, 2008).

Nos últimos anos, estudos relatam a utilização de energias alternativas para a conversão seletiva de biomassa, tais como micro-ondas e ultrassom. Lacerda et al. (2015) avaliou a conversão de materiais lignocelulósicos em plataformas furânicas utilizando líquidos iônicos e dióxido de titânio assistida por micro-ondas em temperaturas inferiores a $200{ }^{\circ} \mathrm{C}$. Cravotto et al. (2009) uniu as micro-ondas com o ultrassom, aumentando a transferência de massa e reduzindo o tempo para extrações de óleos em plantas. Tabasso et al. (2013) propôs a conversão de biomassa a ácido levulínico assistida por micro-ondas, com redução drástica do tempo reacional e com alta seletividade, obtendo até 95\% de conversão.

Considerando a carência de estudos utilizando ultrassom como fonte primária de energia na conversão de biomassa, aliada às condições extremas necessárias nas reações de hidrólise ácida, esse estudo teve como objetivo avaliar a eficiência do ultrassom para conversão de celulose. Buscou-se um processo que proporcione rotas reacionais seletivas para a síntese de compostos furânicos e condições reacionais mais brandas, com ácidos diluídos e temperaturas mais amenas.

\section{MATERIAIS E MÉTODOS}

\subsection{Instrumentação}

Para os experimentos de hidrólise ácida assistida por ultrassom (UAAH), foram utilizados sistemas de ultrassom do tipo sonda, cup horn e banho, de frequências e potências variadas. Para os sistemas de ultrassom do tipo sonda, foram utilizados equipamentos com frequência de $20 \mathrm{kHz}$ e potência de $130 \mathrm{~W}$ ou de $20 \mathrm{kHz}$ e $750 \mathrm{~W}$ (Sonics and Materials Inc., EUA), ambas equipados com sonda de liga de titânio. Também foram utilizados dois sistemas de ultrassom do tipo cup horn (Sonics and Materials Inc., EUA) acoplados a processadores de $20 \mathrm{kHz}$ e $130 \mathrm{~W}$ ou de $20 \mathrm{kHz}$ e $750 \mathrm{~W}$, ambos com cuba de vidro. Para as sondas de ultrassom e para o sistema tipo cup horn a seleção da amplitude de vibração é possível, de 30 a 70\%, sendo este parâmetro também avaliado. Além desses equipamentos, foram utilizados quatro banhos de ultrassom (Elma GmbH \& Co, Singen, Alemanha), os quais foram nomeados de 1 a 4, conforme mostrado na Tabela 1 . 
Tabela 1: Especificações dos banhos de ultrassom utilizados em UAAH.

\begin{tabular}{lcccc}
\hline Banho & Potência efetiva, W & Frequência, kHz & Volume do banho, L & Modelo \\
\hline 1 & 200 & 35 e 130 & 8,6 & TI-H-10 \\
2 & 100 & 25 e 45 & 3,5 & TI-H-5 \\
3 & 330 & 37 e 80 & 12,9 & P120H \\
4 & 330 & 37 e 80 & 9 & P120H \\
\hline
\end{tabular}

As reações de hidrólise foram feitas em provetas de vidro com fundo plano, com $12 \mathrm{~cm}$ de altura, $6 \mathrm{~cm}$ de diâmetro externo e $5 \mathrm{~cm}$ de diâmetro interno. A avaliação/caracterização da seletividade das reações foi feita por cromatografia a líquido de alta eficiência acoplada a espectrometria de massa por tempo de vôo (UPLC-ToF-MS, Xevo G2 Q-Tof, Waters, EUA). Os resíduos de hidrólise foram caracterizados por microscopia eletrônica de varredura (SEM, Sigma 300 VP Carl Zeiss, Inglaterra) e a pureza do produto foi avaliada por ressonância magnética nuclear (NMR, Bruken DPX 400, EUA). Os ensaios sob agitação mecânica (ausência do ultrassom) foram feitos utilizando agitador mecânico (Polytron, Suíça).

\subsection{Reagentes}

Para as reações de hidrólise os seguintes reagentes foram utilizados: ácido nítrico (65\%) (Merck, Alemanha), celulose microcristalina (Sigma-Aldrich, Alemanha) e água purificada pelo sistema Milli-Q ${ }^{\circledR}$ (Ultrapure Water Purification Systems, EUA). Nitrogênio e argônio (White Martins, Brasil), foram utilizados para SEM e como gás de colisão para fonte de ionização em UPLC-Tof-MS. Acetonitrila (Fluka, Steinheim, Alemanha) e metanol (Phillipsburg, EUA) foram utilizados para solubilização e diluição das amostras. Para quantificação foi utilizado um padrão de furfural (99\%, Sigma-Aldrich, Steinheim, Alemanha).

\subsection{Métodos}

Os experimentos foram feitos conforme Santos (2017). Celulose microcristalina (0,5 g) foi transferida para o reator e foram adicionados $20 \mathrm{~mL}$ de solução ácida, diluída à $10 \%$. A mistura foi submetida a aplicação do ultrassom durante $60 \mathrm{~min}$, com a temperatura de $50{ }^{\circ} \mathrm{C}$, em todos os sistemas de ultrassom avaliados. Após os experimentos preliminares, com os resultados satisfatórios obtidos e com auxílio de um estudo da potência liberada por calorimetria, o sistema cup horn foi selecionado para continuação do estudo. Em seguida, os parâmetros avaliados foram: concentração de ácido $\left(4,6\right.$ e $\left.8 \mathrm{~mol} \mathrm{~L}^{-1} \mathrm{HNO}_{3}\right)$, temperatura da reação $\left(30,50\right.$ e $\left.70{ }^{\circ} \mathrm{C}\right)$ e amplitude do ultrassom (30, 50 e 70\%). Paralelamente, a eficiência de conversão foi comparada com a obtida para o processo sob agitação mecânica (100 rpm, $50{ }^{\circ} \mathrm{C}$ e $\left.60 \mathrm{~min}\right)$. Após a reação, os extratos foram filtrados e diluídos com metanol (1:10) para posterior determinação por UPLC-ToF-MS e a pureza do produto foi avaliada por NMR de ${ }^{1} \mathrm{H} \mathrm{e}{ }^{13} \mathrm{C}$. O resíduo de celulose obtido após UAAH foi seco e caracterizado por SEM. 


\section{RESULTADOS E DISCUSSÃO}

Neste estudo, a intensidade de potência foi avaliada pelo método calorimétrico (Kimura et al., 1996), baseado na variação da temperatura durante a aplicação do ultrassom, na massa de celulose e no calor específico da solução. A intensidade de potência observada nos banhos de ultrassom foi na faixa de 1 a $2,5 \mathrm{~W} \mathrm{dm}^{-3}$ e para os sistemas cup horn e sonda foi de 3 e 14 $\mathrm{W} \mathrm{dm}{ }^{-3}$, respectivamente. Para a reação feita sob agitação mecânica, a potência liberada foi menor, de cerca de $1 \mathrm{~W} \mathrm{dm}^{-3}$.

Apesar da sonda fornecer a maior intensidade de potência dentre os sistemas avaliados, não foi constatada a presença de furfural nos extratos provenientes deste sistema. É possível que um incremento na intensidade de potência liberada favoreça a formação de radicais que interferem no curso e na seletividade da reação, além da maior energia liberada. A presença de furfural também não foi detectada para os experimentos utilizando os banhos de ultrassom ou sob agitação mecânica. Para esses casos, da mesma forma para os experimentos utilizando a sonda, apenas fragmentos de monômeros de carboidratos foram identificados. Por outro lado, no sistema cup horn a formação de furfural foi detectada e esse sistema foi selecionado para os experimentos seguintes. Além disso, cabe ressaltar que em todos os experimentos, restou um resíduo de celulose microcristalina degradada ao final da UAAH.

Em seguida, foi feita a avaliação da concentração da solução ácida para hidrólise. Os resultados obtidos para $\mathrm{HNO}_{3}$ em diferentes concentrações estão mostrados na Tabela 2.

Tabela 2: Conversão de celulose a furfural por UAAH

\begin{tabular}{cc}
\hline Concentração de $\mathrm{HNO}_{3}, \mathrm{~mol} \mathrm{~L}^{-1}$ & Conversão, \% \\
\hline 4 & 78 \\
6 & 55 \\
8 & 33 \\
\hline
\end{tabular}

Foi observado decréscimo na conversão de furfural com aumento da concentração ácida, o que pode ser justificado pela oxidação do produto formado, levando a formação de compostos furânicos de menor peso molecular.

Em relação à influência da temperatura na conversão de celulose, foram feitos experimentos com temperaturas de 30 a $70{ }^{\circ} \mathrm{C}$, sendo obtida a maior seletividade na temperatura de $30{ }^{\circ} \mathrm{C}$. Em temperaturas superiores, foi observada a degradação da celulose, porém sem seletividade para produção de furanos.

Uma vez otimizada a concentração ácida $\left(4 \mathrm{~mol} \mathrm{~L}^{-1}\right)$ e a temperatura reacional $\left(30{ }^{\circ} \mathrm{C}\right)$, foram feitos experimentos avaliando diferentes amplitudes de ultrassom (30, 50 e 70\%). Não foi obtida a formação de furfural quando foi utilizada amplitude de $30 \%$. Nos ensaios com amplitude de 50 e 70\%, foi observada intensidade similar de sinal. Assim, a condição otimizada foi de: $0,5 \mathrm{~g}$ de celulose e $20 \mathrm{~mL}$ de $\mathrm{HNO}_{3} 4 \mathrm{~mol} \mathrm{~L}^{-1}$ em sistema cup horn, operando por 60 minutos, com amplitude de $50 \%$ e temperatura de $30{ }^{\circ} \mathrm{C}$. 
A celulose microcristalina original (matéria-prima) e o resíduo após a UAAH foram caracterizados por SEM para avaliar as consequências do tratamento com ultrassom. A Figura 1 mostra as imagens obtidas para a celulose: (a) antes do tratamento e (b) após UAAH.

Figura 1: Microscopia eletrônica de varredura para (a) celulose microcristalina e para (b) resíduo após UAAH.
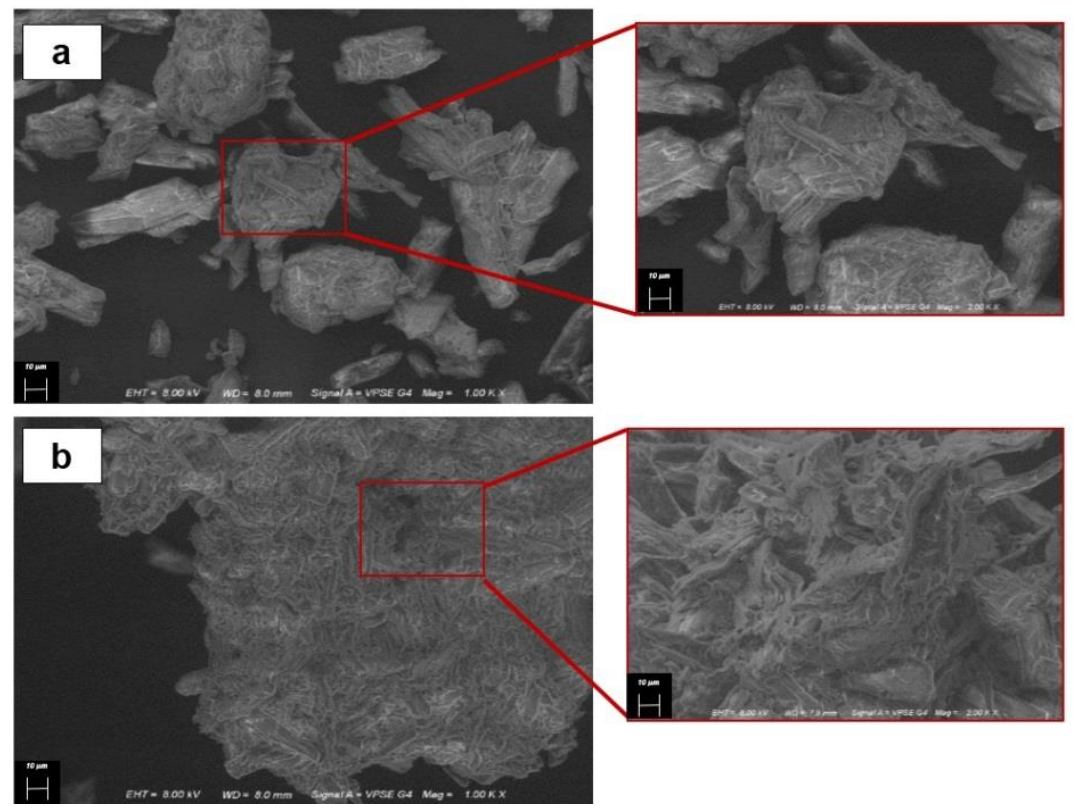

A imagem da figura 1(a) mostra uma estrutura organizada, caracterizada por morfologia lamelar. Já na figura 1(b), após o tratamento, é possível observar que ocorre uma desorganização dessa estrutura, evidenciando a ruptura nas cadeias poliméricas da celulose, deixando os monômeros glicosídicos expostos ao ataque ácido, o que possibilita contato mais efetivo e um consequente incremento na conversão à furfural.

\section{CONCLUSÃO}

Neste estudo foi investigada a conversão de celulose em furfural, um importante produto químico, através do uso do ultrassom como fonte de energia alternativa. Foram obtidos rendimentos de até $78 \%$ de conversão à furfural a partir de celulose, empregando temperaturas amenas $\left(30{ }^{\circ} \mathrm{C}\right)$ e soluções ácidas diluídas $\left(\mathrm{HNO}_{3} 4 \mathrm{~mol} \mathrm{~L}^{-1}\right)$, em tempos relativamente curtos (60 min) com uso de ultrassom em sistema do tipo cup horn. A hidrólise ácida assistida por ultrassom (UAAH) se mostrou útil para a conversão de celulose, com tempos e temperaturas de reação inferiores, promovendo ganhos de energia. 


\section{REFERÊNCIAS BIBLIOGRÁFICAS}

BARBOSA, C. Biomassa para energia. UNICAMP, 2008.

CRAVOTTO, G.; BOFFA, L.; MANTEGNA, S.; PEREGO, P.; AVOGADRO, M.; CINTAS, P. Improved extraction of vegetable oils under high-intensity ultrasound and/or microwaves, Ultrasonic. Sonochem., v. 15, p. 898-902, 2008.

KIMURA T. et al. Standardization of ultrasonic power for sonochemical reaction, Ultrasonic. Sonochem., v. 3, p. 157-161, 1996.

LACERDA, V.S. et al. A kinetic study on microwave-assisted conversion of cellulose and lignocellulosic waste into hydroxymethylfurfuralfurfural. Biores. Tec., v. 180, p. 88-96, 2015.

MOTHÉ, C.G.; MIRANDA I.C. Aproveitamento energético e caracterização de resíduos de biomassa. Publit, 2010.

ROCHA, M.H. Avaliação técnica de biorrefinarias para a produção de biocombustíveis líquidos e eletricidade através da gaseificação de biomassa. Tese de Doutorado. Programa de Pós-graduação em Engenharia Mecânica. Universidade Federal de Itajuba, Itajubá, 2015.

RODRIGUES, J.A.R. Do engenho a biorrefinaria: a usina de açucar como empreendimento industrial para a geração de produtos bioquímicos e combustíveis. Quim. Nova, v. 34, p.12421254, 2011.

SANTOS, D. Dissertação de mestrado (em andamento). Programa de Pós-Graduação em Engenharia Química. Universidade Federal de Santa Maria, Santa Maria, conclusão prevista para 2017.

SILVEIRA, M.S. Aproveitamento de cascas de coco verde para a produção de briquete em Salvador-BA. Dissertação de Mestrado. Programa de Pós-Graduação em Gerenciamento e Tecnologias Ambientais no Processo Produtivo. Universidade Federal da Bahia, Salvador, 2008.

TABASSO S. et al. Microwave-assisted flash conversion of non-edible polysaccharides and post-harvest tomato plant waste to levulinic acid. Green Chem., v. 16, p. 73-76, 2014. 